08

\title{
Зависимость электронной структуры графеновой наноленты от концентрации адсорбированных частиц
}

\author{
() С.Ю. Давыдов \\ Физико-технический институт им. А.Ф. Иофрфе РАН, Санкт-Петербург, Россия \\ E-mail: Sergei_Davydov@mail.ru
}

Поступило в Редакцию 18 марта 2020 г.

В окончательной редакции 26 марта 2020 г.

Принято к публикации 26 марта 2020 г.

\begin{abstract}
Рассмотрено влияние косвенного обмена адсорбированных на графеновой наноленте частиц на ширину наведенной энергетической щели и эффективную массу носителей. Получены аналитические зависимости этих характеристик от концентрации адчастиц энергии связи адсорбат-подложка и энергии уровня адсорбируемой частицы.
\end{abstract}

Ключевые слова: нанолента графена с зигзагообразными кромками, косвенное взаимодействие адчастиц, энергетическая щель, эффективная масса.

DOI: 10.21883/PJTF.2020.13.49581.18292

Уникальные свойства графена, позволяющие использовать его в экстремальных условиях, делают этот материал весьма перспективным для наноэлектроники [1-3]. Определенным препятствием на этом пути является отсутствие энергетической щели в спектре идеального (беспримесного, бездефектного, механически ненапряженного) бесконечного свободного (т.е. в отсутствие подложки) листа графена. Для того чтобы создать щель, нужно нарушить условия идеальности $[4,5]$. Одним из способов является адсорбция чужеродных атомов [6-8]. Подавляющее число теоретических работ по адсорбции на графене выполнено в рамках DFT (density functional theory) [6-10]. Здесь мы рассмотрим взаимодействующие между собой адчастицы при различных их концентрациях, воспользовавшись для этого методом функций Грина $[11,12]$.

Как известно [12,13], между адатомами имеется три основных канала взаимодействия. Первый канал - это электростатическое отталкивание параллельных диполей, образованных адатомами и их изображениями в подложке при условии перехода заряда. Второй канал прямой обмен, т.е. непосредственный переход электронов между адатомами при их высокой концентрации, когда имеет место перекрытие орбиталей ближайших соседей. И наконец, третий канал предстваляет собой косвенный (непрямой) обмен адатомов электронами через зонные состояния подложки. Косвенный обмен, являясь, как и дипольное взаимодействие, дальнодействующим, проявляется уже при малых покрытиях. Влияние дипольдипольного отталкивания и косвенного обмена на заряд адатомов рассматривалось нами в работе [14]. Роль прямого обмена легко понять из рассмотрения малых атомных кластеров на поверхности графена [14,15].

В работах $[14,15]$ показано, что диполь-дипольное отталкивание и прямой обмен не приводят к возникновению щели. Косвенный обмен наводит в электронном спектре графенового листа щель $\Delta_{L}=\sqrt{\varepsilon_{a}^{2}+4 \Theta V^{2}}[16]$, где $\varepsilon_{a}$ - энергия уровня адсорбированной частицы, отсчитываемая от точки Дирака; $V-$ усредненный по зоне Бриллюэна графена матричный элемент связи адсорбат-подложка; $\Theta=N_{a} / N_{\mathrm{ML}}-$ степень покрытия поверхности адсорбатом $\left(N_{a} \neq 0\right.$ и $N_{\mathrm{ML}}$ - концентрации адатомов в субмонослое и монослое соответственно, $0<\Theta \leq 1)$.

Для описания графеновой наноленты с зигзагообразными кромками (ZNR) воспользуемся двухцепочечной моделью [17] (см. рис. 1 в [17]), в рамках которой дисперсия электронов для свободной от адатомов ZNR дается выражением

$$
\varepsilon_{p}(k)= \pm t[\sqrt{1+4 \Phi(k)} \pm 1] / 2
$$

где $\Phi(k)=4 \cos ^{2}(k a), a-$ расстояние между ближайшими соседями, $|k| \leq \pi / 2 a$ - волновой вектор для движения электрона вдоль цепочек. За нуль энергии вновь принята энергия точки Дирака графена, индекс $p$ нумерует зоны (нумерация зон идет в сторону убывания энергии, так что $\varepsilon_{1}(k)=-\varepsilon_{4}(k)$ и $\left.\varepsilon_{2}(k)=-\varepsilon_{3}(k)\right)$. Как следует из (1), щель в спектре невозмущенной наноленты отсутствует, так как при $|k|=\pi / 2 a$ (граница зоны Бриллюэна) имеем $\varepsilon_{2}(k)=\varepsilon_{3}(k)=0$ (см. рис. 2 в [17]). При $|k| \rightarrow \pi / 2 a$ имеем дисперсионные соотношения $\varepsilon_{1,4}(q) \approx \pm t\left(1+4 q^{2} a^{2}\right)$ и $\varepsilon_{2,3}(q) \approx \pm 4 t q^{2} a^{2}$, где $q=k-2 \pi / a$. Низкоэнергетическим зонам $\varepsilon_{2,3}(q)$ отвечает приведенная эффективная масса $m^{*}=m_{e} / m_{0}=-m_{h} / m_{0}=\hbar^{2} / 8 t a^{2} m_{0} \approx 0.17$, где $t=2.8 \mathrm{eV}, a=1.42 \AA, m_{e(h)}$ - эффективная масса электронов (дырок), $m_{0}$ - масса свободного электрона.

Перейдем теперь к задаче об адсорбции. Обобщая результаты работы [16], функции Грина зон ZNR и 

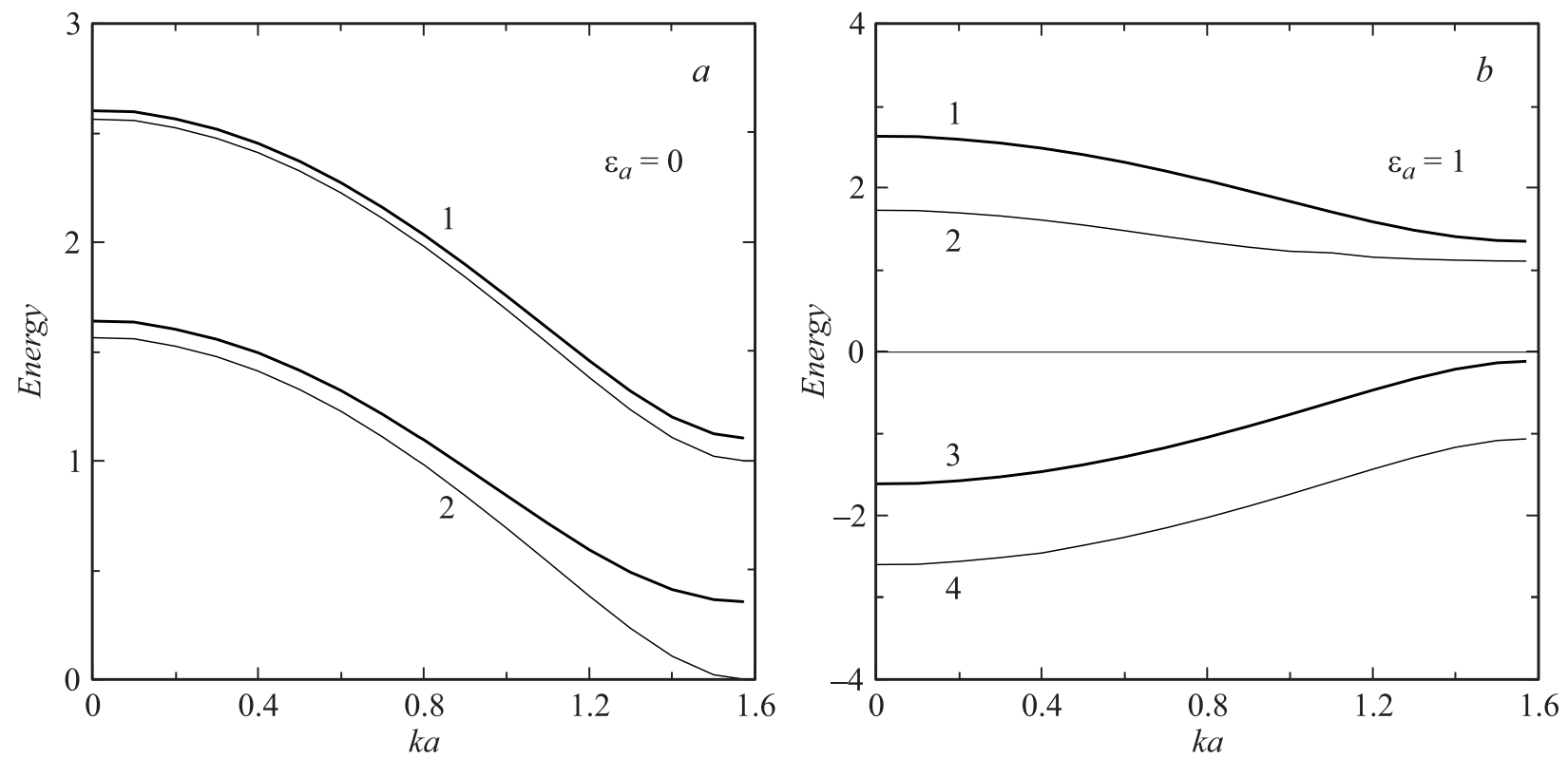

Рис. 1. Энергетические зоны $E_{p}(k)$ (жирные линии): $\varepsilon_{a}=0(a)$ и $1(b) ; 4 \Theta V^{2}=0.5$. На части $a$ тонкими линиями показаны зоны $\varepsilon_{p}(k)$ в отсутствие адсорбции. За нуль энергии принята точка Дирака, все энергетические величины измеряются в единицах $t$. Цифры соответствуют зонному индексу $p$.

адатомов можно представить в виде

$$
\begin{gathered}
G_{\mathrm{ZNR}}(\omega)=\frac{1}{N} \sum_{k, p} \frac{\omega-\varepsilon_{a}}{D_{p}(\omega, k)}, \\
G_{a}(\omega)=\frac{1}{N} \sum_{k, p} \frac{\omega-\varepsilon_{k}}{D_{p}(\omega, k)},
\end{gathered}
$$

где $D_{p}(\omega, k)=\left(\omega-\varepsilon_{p}(k)\right)\left(\omega-\varepsilon_{a}\right)-\Theta V^{2}, \omega$ - энергетическая переменная, $N$ - число атомов ZNR (заметим, что функции Грина (2) соответствуют усредненному бесструктурному адслою). Спектр системы $E_{p}(k)$ находим из уравнения $D_{p}(\omega, k)=0$ :

$$
\begin{aligned}
& E_{1}(k)=\frac{1}{2}\left(\varepsilon_{a}+\varepsilon_{1}(k)+\sqrt{\left(\varepsilon_{a}-\varepsilon_{1}(k)\right)^{2}+4 \Theta V^{2}}\right), \\
& E_{2}(k)=\frac{1}{2}\left(\varepsilon_{a}+\varepsilon_{2}(k)+\sqrt{\left(\varepsilon_{a}-\varepsilon_{2}(k)\right)^{2}+4 \Theta V^{2}}\right), \\
& E_{3}(k)=\frac{1}{2}\left(\varepsilon_{a}-\varepsilon_{2}(k)-\sqrt{\left(\varepsilon_{a}+\varepsilon_{2}(k)\right)^{2}+4 \Theta V^{2}}\right), \\
& E_{4}(k)=\frac{1}{2}\left(\varepsilon_{a}-\varepsilon_{1}(k)-\sqrt{\left(\varepsilon_{a}+\varepsilon_{1}(k)\right)^{2}+4 \Theta V^{2}}\right),
\end{aligned}
$$

где мы учли соотношения $\varepsilon_{1}(k)=-\varepsilon_{4}(k)$ и $\varepsilon_{2}(k)=-\varepsilon_{3}(k) \quad\left(\right.$ при $\quad \varepsilon_{a}=0 \quad$ те $\quad$ же соотношения справедливы и для $\left.E_{p}(k)\right)$. На границе зоны Бриллюэна низкоэнергетические зоны $E_{2}(k)$ и $E_{3}(k)$ разделены щелью $\Delta_{0} \equiv \Delta\left(\varepsilon_{a}=0\right)=2 V \sqrt{\Theta}$ (рис. $\left.1, a\right)$. В случае $\varepsilon_{a}>0$ все зоны $E_{p}(k)$ смещаются вверх по шкале энергий по сравнению со случаем $\varepsilon_{a}=0$, а нумерация зон остается той же, что и при $\varepsilon_{a}=0$ (рис. $\left.1, b\right)$.
В случае $\varepsilon_{a}<0$ зоны $E_{p}(k)$ сдвигаются вниз относительно случая $\varepsilon_{a}=0$. При этом (по сравнению со случаем $\left.\varepsilon_{a}>0\right)$ имеют место следующие равенства: $E_{1}\left(\varepsilon_{0}>0\right)=-E_{4}\left(\varepsilon_{0}<0\right), \quad E_{2}\left(\varepsilon_{0}>0\right)=-E_{3}\left(\varepsilon_{0}<0\right)$. Щель между зонами $E_{2}(k)$ и $E_{3}(k)$ в общем случае равна

$$
\Delta=\sqrt{\varepsilon_{a}^{2}+4 \Theta V^{2}},
$$

что совпадает с шириной щели $\Delta_{L}$, наводимой адсорбцией в графеновом листе. Если $\varepsilon_{a}^{2}<4 V^{2}$, то при $\Theta<\bar{\Theta}=\varepsilon_{a}^{2} / 4 V^{2}$ имеем $\Delta \sim\left|\varepsilon_{a}\right|\left(1+2 \Theta V^{2} / \varepsilon_{a}^{2}\right)$, тогда как при условии $1>\Theta>\bar{\Theta}=\varepsilon_{a}^{2} / 4 V^{2}$ получаем $\Delta \sim 2 V \sqrt{\Theta}\left(1+\varepsilon_{a}^{2} / 8 \Theta V^{2}\right)$.

Если в окрестности щели, наведенной адсорбцией в бесконечном листе графена, дисперсия остается линейной [15], то в случае ZNR ситуация другая. Действительно, вблизи границы зоны Бриллюэна имеем $E_{2}(q) \approx\left(\varepsilon_{a}+\Delta\right) / 2+2 t q^{2} a^{2}\left(1-\varepsilon_{a} / \Delta\right)$ и $E_{3}(q) \approx\left(\varepsilon_{a}-\Delta\right) / 2-2 t q^{2} a^{2}\left(1+\varepsilon_{a} / \Delta\right), \quad$ так что область щели соответствует энергетическому интервалу $\left[\left(\varepsilon_{a}-\Delta\right) / 2,\left(\varepsilon_{a}+\Delta\right) / 2\right]$. Эффективные массы зон $E_{2}(k)$ и $E_{3}(k)$ равны

$$
m_{e, h}^{*}= \pm \frac{\hbar^{2}}{4 t a^{2} m_{0} \beta_{e, h}}, \quad \beta_{e, h}=1 \mp \varepsilon_{a} / \Delta,
$$

где $\beta_{e, h}>0$, так как $0 \leq\left|\varepsilon_{a}\right| / \Delta<1$. При $\varepsilon_{0}=0$ получаем $m^{*}=m_{e}^{*}=\left|m_{h}^{*}\right| \approx 0.34$, что вдвое превосходит эффективную массу свободной ZNR. Зависимости ширины щели $\Delta$ и эффективных масс $m_{e}^{*}$ и $\left|m_{h}^{*}\right|$ представлены на рис. 2. Резкий рост $m_{e}^{*}$ при $\varepsilon_{a}^{2} \gg 4 \Theta V^{2} \quad\left(\varepsilon_{a}>0\right)$ отвечает уплощению зоны проводимости: $E_{2}(k) \rightarrow \varepsilon_{a}$. Аналогичная ситуация при $\varepsilon_{a}<0$ имеет место для $\left|m_{h}^{*}\right|$ : 

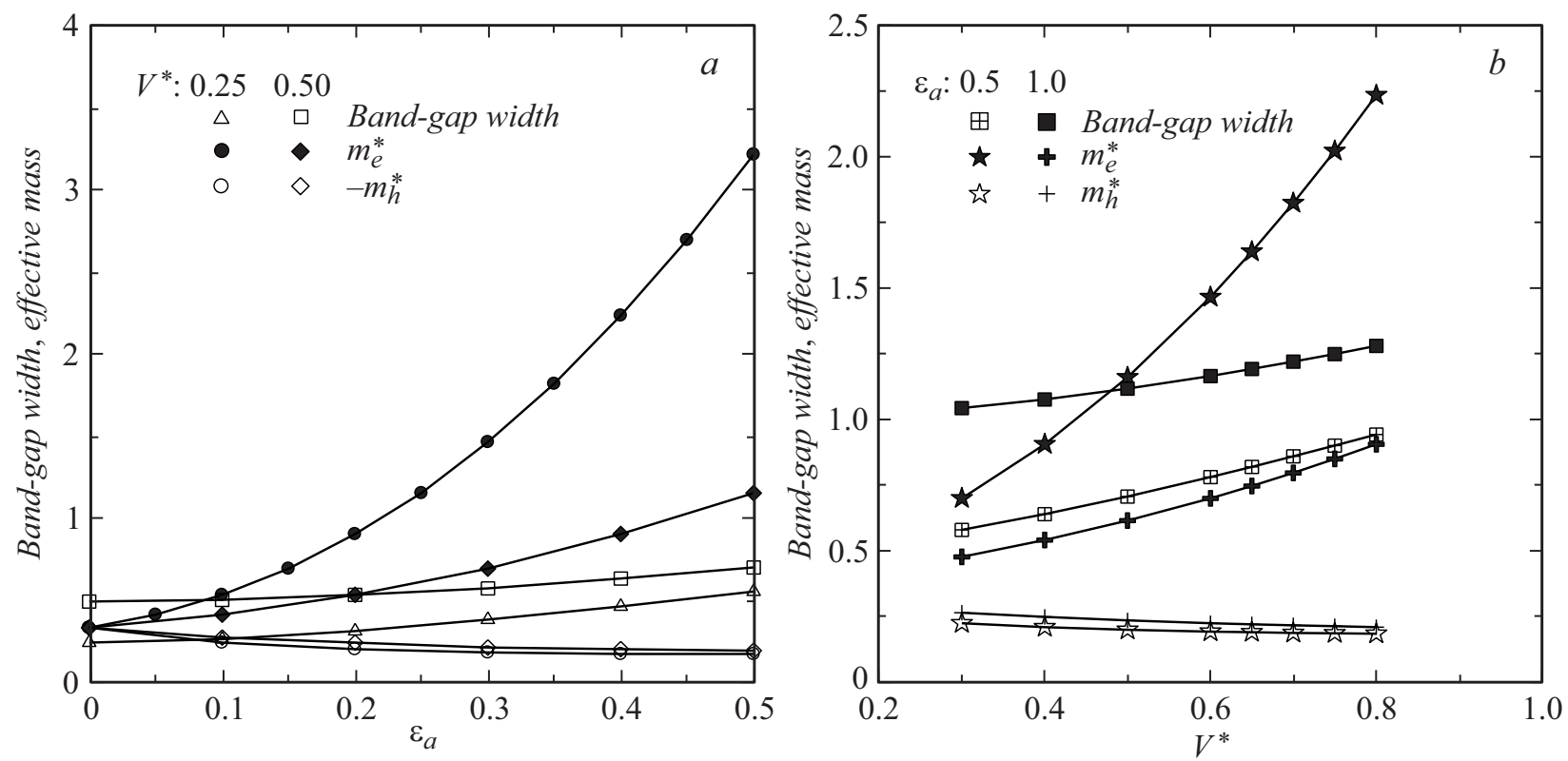

Рис. 2. Зависимости ширины щели $\Delta$ и эффективных масс электронов $m_{e}^{*}$ и дырок $\left|m_{h}^{*}\right|=-m_{h}^{*}$ от энергии уровня адчастицы $\varepsilon_{a}(a)$ и параметра взаимодействия $V^{*}=2 V \sqrt{\Theta}(b)$. Приведены данные только для $\varepsilon_{a}>0$, так как $\Delta\left(-\varepsilon_{a}\right)=\Delta\left(\varepsilon_{a}\right)$ и $m_{e}^{*}\left(-\varepsilon_{a}\right)=\left|m_{h}^{*}\left(\varepsilon_{a}\right)\right|$. Все энергетические параметры измеряются в единицах $t$.

$E_{3}(k) \rightarrow \varepsilon_{a}<0$. При этом значения $m_{h}^{*}$ в первом и $m_{e}^{*}$ во втором случае стремятся к эффективным массам свободной ZNR. Эти тенденции можно усмотреть уже из формул (2) в пределе $\Theta V^{2} \rightarrow 0$. Отметим, что уплощение зон наблюдалось и в задаче о декорировании ZNR [17].

Сопоставим полученные нами зависимости с некоторыми результатами расчетов [18], где изучалось влияние концентрации адчастиц в упорядоченных и разупорядоченных слоях на электронный спектр и кондактанс графеновой ZNR. Первый вывод работы [18] гласит: при $\Theta=1$ и $\varepsilon_{a}=0$ ширина щели $\Delta \propto V^{2}$, тогда как, согласно нашей модели, $\Delta_{0}(\Theta=1)=2 V$ (здесь и далее мы заменяем обозначения $\varepsilon_{\alpha}, n_{i}, \gamma$ и $E_{g}$ работы [18] на $\varepsilon_{a}, \Theta, V$ и $\left.\Delta\right)$. Второй вывод относится к малым $\Theta \ll 1$ и большим $\Theta=1-\vartheta(\vartheta \ll 1)$ степеням покрытия при $\varepsilon_{a}=0$. В первом случае в [18] констатируется увеличение ширины щели с ростом $\Theta$, во втором - уменьшение ширины щели при $\vartheta \rightarrow 0$. В нашей модели $\Delta_{0}=2 V \sqrt{\Theta}$, т.е. ширина щели увеличивается во всем диапазоне изменения степени покрытия. И наконец, третий вывод [18] относится к разупорядоченному адсорбату: при малых $\Theta$ щель отсутствует, но с ростом $\Theta$ появляется. Для проверки справедливости этого утверждения будем, как и в [18], полагать, что значения $\varepsilon_{a}$ равномерно распределены в интервале $\left[\varepsilon_{a}-w, \varepsilon_{a}+w\right]$. Тогда среднее значение ширины щели равно $\bar{\Delta}=(2 w)^{-1} \int_{-w}^{w} \sqrt{\varepsilon_{a}^{2}+4 \Theta V^{2}} d \varepsilon_{a}$, откуда

$$
\bar{\Delta}=\frac{2 \Theta V^{2}}{w} \ln \left|\frac{w+2 V \sqrt{\Theta}}{w-2 V \sqrt{\Theta}}\right| \text {. }
$$

При $\Theta \ll(w / 2 V)^{2}$ получаем $\bar{\Delta} \approx 8 \Theta^{3 / 2} V^{3} / w^{2}$, так что в линейном приближении по $\Theta$ щель отсутствует. К сожалению, сделать аналогичное сравнение для эффективных масс не представляется возможным, так как нам неизвестны соответствующие экспериментальные или теоретические данные.

Таким образом, в рамках простой модели нам удалось в аналитическом виде представить влияние косвенного обмена между адчастицами на ширину щели и эффективную массу носителей в наноленте.

\section{Конфликт интересов}

Автор заявляет, что у него нет конфликта интересов.

\section{Список литературы}

[1] Wu Y.H., Yu T., Shen Z.X. // Appl. Phys. Rev. 2010. V. 108. N 7. P. 071301.

[2] Meunier V., Souza Filho A.G., Barros E.B., Dresselhaus M.S. // Rev. Mod. Phys. 2016. V. 88. N 2. P. 025005.

[3] Sang M., Shin J., Kim K., Yu K.J. // Nanomaterials. 2019. V. 9. N 3. P. 374-406.

[4] Kuila T., Bose S., Mishra A.K., Khanra P., Kim N.H., Lee J.H. // Prog. Mater. Sci. 2012. V. 57. N 7. P. 1061-1105.

[5] Xu X., Liu C., Sun Z., Cao T., Zhang Z., Wang E., Liu Z., Liu K. // Chem. Soc. Rev. 2018. V. 47. N 9. P. 3059-3099.

[6] Kong L., Enders A., Rahman T.S., Dowben P.A. // J. Phys.: Condens. Matter. 2014. V. 26. N 44. P. 443001.

[7] Ersan G., Apul O.G., Perreault F., Karanfil T. // Water Res. 2017. V. 126. P. 385-398. 
[8] Murata Y., Calzolari A., Heun S. // J. Phys. Chem. C. 2018. V. 122. N 21. P. 1591-1597.

[9] Nakada K., Ishii A. DFT calculation for adatom adsorption on graphene // Graphene simulation / Ed. J.R. Gong. InTechOpen, 2011. Available: http://www.intechopen.com/books/graphene-simulation/dftcalculation-for-adatom-adsorption-on-graphene

[10] Dimakis N., Salas I., Gonzalez L., Vadodaria O., Ruiz K., Bhatti M.I. // Molecules. 2019. V. 24. N 4. P. 754-781.

[11] Davison S.G., Sulston K.W. Green-function theory of chemisorption. Springer Netherlands, 2006. 211 p.

[12] Давыдов С.Ю. Теория адсорбции: метод модельных гамильтонианов. СПб.: Изд-во СПбГЭТУ „ЛЭТИ“, 2013. 235 c.; twirpx.com/file/1596114/

[13] Браун О.М., Медведев В.К. // УФН. 1989. Т. 157. № 4. C. $631-666$.

[14] Давыдов С.Ю. // Письма в ЖТФ. 2014. Т. 40. В. 13. C. $52-57$.

[15] Давыдов С.Ю. // Письма в ЖТФ. 2012. Т. 38. В. 15. C. $25-33$.

[16] Давыдов С.Ю. // ФТП. 2012. Т. 46. В. 2. С. 204-209.

[17] Давыдов С.Ю. // ФТТ. 2019. Т. 61. В. 3. С. 610-617.

[18] Chen L., Ouyang F., Ma S., Fang T.-F., Guo A.-M., Sun Q.-F. arXiv: $1912.07986 \mathrm{v} 1$ 